\title{
The Dark Energy Spectrometer-A Potential Multi-Fiber Instrument for the Blanco 4-meter Telescope
}

\author{
J. L. Marshall ${ }^{1}$, Stephen M. Kent ${ }^{2,3}$, H. Thomas Diehl' ${ }^{2}$, Brenna Flaugher ${ }^{2}$, Joshua Frieman ${ }^{2,3}$, \\ Richard G. Kron ${ }^{2,3}$, D. L. DePoy ${ }^{1}$, Matthew Colless ${ }^{4}$, Will Saunders ${ }^{4}$, Greg A. Smith ${ }^{4}$, Ofer Lahav ${ }^{5}$, \\ Filipe Abdalla ${ }^{5}$, David Brooks ${ }^{5}$, Peter Doel ${ }^{5}$, Donnacha Kirk ${ }^{5}$, James Annis ${ }^{2}$, Huan Lin ${ }^{2}$, John P. \\ Marriner $^{2}$, Stephanie Jouvel ${ }^{6}$, Michael D. Seiffert ${ }^{7}$ \\ ${ }^{1}$ Department of Physics and Astronomy, Texas A\&M University, 4242 TAMU, College Station, TX, \\ $77843-4242$ USA \\ ${ }^{2}$ Fermi National Accelerator Laboratory, Box 500, Batavia IL, 60510 USA \\ ${ }^{3}$ University of Chicago, 5640 S. Ellis Avenue, Chicago IL 60637 USA \\ ${ }^{4}$ Australian Astronomical Observatory, PO Box 296, Epping NSW, 1710 Australia \\ ${ }^{5}$ University College London, Gower Street, London WC1E 6BT UK \\ ${ }^{6}$ Institut de Ciències de 1'Espai (IEEC-CSIC), E-08193 Bellaterra (Barcelona) Spain \\ ${ }^{7}$ Jet Propulsion Laboratory, California Institute of Technology, 4800 Oak Grove Drive, Pasadena, \\ CA 91109 USA
}

\begin{abstract}
We describe the preliminary design of the Dark Energy Spectrometer (DESpec), a fiber-fed spectroscopic instrument concept for the Blanco 4-meter telescope at Cerro Tololo Inter-American Observatory (CTIO). DESpec would take advantage of the infrastructure recently deployed for the Dark Energy Camera (DECam). DESpec would be mounted in the new DECam prime focus cage, would be interchangeable with DECam, would share the DECam optical corrector, and would feature a focal plane with $\sim 4000$ robotically positioned optical fibers feeding multiple high-throughput spectrometers. The instrument would have a field of view of 3.8 square degrees, a wavelength range of approximately $500<\lambda<1000 \mathrm{~nm}$, and a spectral resolution of $\mathrm{R} \sim 3000$. DESpec would provide a powerful spectroscopic follow-up system for sources in the Southern hemisphere discovered by the Dark Energy Survey and LSST.
\end{abstract}

Keywords: dark energy, fiber spectrometers, wide field spectroscopy

\section{INTRODUCTION}

The discovery of dark energy is arguably the most important cosmological discovery in the past thirty years. New insights into the nature of dark energy will come soon from the Dark Energy Survey (DES), a deep, wide, multi-band imaging survey, spanning 525 nights over five years beginning in late 2012, that will use the new 570-Megapixel Dark Energy Camera (DECam) ${ }^{[1],[2]}$ on the 4 meter Blanco telescope at CTIO. DES, in partnership with ESO's near-infrared VISTA Hemisphere Survey, will provide imaging of $\sim 300$ million galaxies in eight optical-NIR filters (grizY + JHK) over 5000 square degrees as well as $\sim 4000$ well-measured Supernova Ia light curves in a time-domain survey of 30 square degrees. With this survey, DES will probe dark energy using four techniques: the clustering of galaxies on large scales, including baryon acoustic oscillations (BAO); the abundance of massive galaxy clusters; weak gravitational

Ground-based and Airborne Instrumentation for Astronomy IV, edited by lan S. McLean, Suzanne K. Ramsay, Hideki Takami, Proc. of SPIE Vol. 8446, 844656 · C 2012 SPIE · CCC code: 0277-786X/12/\$18 · doi: 10.1117/12.926451 
lensing distortions of the images of distant galaxies; and Type Ia Supernova distances. In the language of the Dark Energy Task Force (DETF) report ${ }^{[3]}$, DES is a "Stage III" experiment that will make a substantial step forward in constraining the properties of dark energy.

As a result of the success to date of the DES project, we have conceived of a conceptual next-generation dark energy project. The project would provide enhanced constraints on dark energy, enable radial baryon acoustic oscillations and redshift-space distortion measurements, provide constraints on neutrino masses, and provide spectroscopic redshifts for thousands of objects in the Southern hemisphere. The observations would be made possible by a new multi-fiber optical instrument for the CTIO Blanco 4-meter telescope called the Dark Energy Spectrometer (DESpec). Together with DES, this project would have the science reach of a next-generation, DETF Stage IV project. DESpec would provide a powerful spectroscopic follow-up system for the millions of Southern hemisphere sources discovered by DES (and later LSST), greatly enhancing the scientific return of both of these large-scale surveys. The DESpec project is comparable to and would complement the proposed BigBOSS survey in the Northern hemisphere ${ }^{[4]}$; by roughly doubling the survey volume, DESpec+BigBOSS together would enable even stronger DE constraints.

Conceived as an upgrade to DECam once the Dark Energy Survey is complete, DESpec would take advantage of the recently deployed DECam infrastructure at CTIO. The conceptual DESpec instrument is a new multi-fiber spectrometer that would use the same mechanical structure and most of the corrector optics of DECam. The DESpec detectors and associated readout electronics would be identical to those used for DECam. The instrument could consist of $\sim 10$ singlearm optical-fiber spectrographs fed by a robotic, multi-fiber positioner at the prime focus of the CTIO $4 \mathrm{~m}$. In one of the initial conceptual designs presented here, DESpec would operate over the wavelength range $550<\lambda<950 \mathrm{~nm}$, have moderate resolution $(\mathrm{R} \sim 3000$ ), and be able to observe $\sim 4000$ targets simultaneously. Other instrument concepts are also being explored and the final instrument design will depend on the exact project science goals, constraints based on community needs and available telescope time, and other factors. DESpec will be required to be interchangeable with DECam in a reasonably short time.

In addition to its dark energy goals, as a community instrument DESpec would enable a wide array of spectroscopic surveys that would afford opportunities for discoveries in stellar structure and evolution, nearby galaxies, galaxy evolution, the structure of galaxy clusters, and beyond. Given the large number of fibers, and building on the experience of the Sloan Digital Sky Survey, one could expect to optimize efficiency by conducting several programs in parallel.

In this paper we describe the modifications and new designs required to transform DECam into DESpec. In particular, we describe notional designs of an optical corrector and atmospheric dispersion compensator, fiber positioner, optical fibers, and unit spectrographs and detectors. We also briefly describe how the instrument could be interchanged with the DECam instrument, and how DESpec might be mounted on the CTIO Blanco 4m telescope. These initial designs serve as a proof of concept for the DESpec instrument, and demonstrate that it is possible to build and operate such an instrument on the Blanco telescope; i.e., the detailed designs will necessarily evolve as the science case for the project becomes more mature.

\section{DESPEC OPTICAL CORRECTOR AND ADC}

The DESpec optical corrector will benefit from previous experience with the DECam optical corrector; in fact we plan to share most of the elements of the DECam optical corrector with the DESpec instrument. The DECam optical corrector was designed to make excellent images in individual filter bandpasses, but refocus was allowed, and lateral chromatic aberrations were controlled only within each bandpass. The 5-element DECam corrector optics produce a flat focal plane with a clear aperture of diameter $\mathrm{D} \sim 450 \mathrm{~mm}$ in the focal plane. It has an $f / 2.9$ beam that has 3.8 degrees of nontelecentricity at the edge of the focal plane. It also uses a filter-changer with $13 \mathrm{~mm}$-thick filters. The DECam lenses are called "C1" (the largest, furthest from the focal plane) through "C5." C5 is the final DECam optical element and is also the imager Dewar window: it travels with DECam when it is removed from the telescope. C1 through C4 would remain in the prime focus cage during an instrument swap of DECam with DESpec. As of this writing (June 2012), the assembled and aligned DECam corrector optics are in the process of being installed on the telescope. 


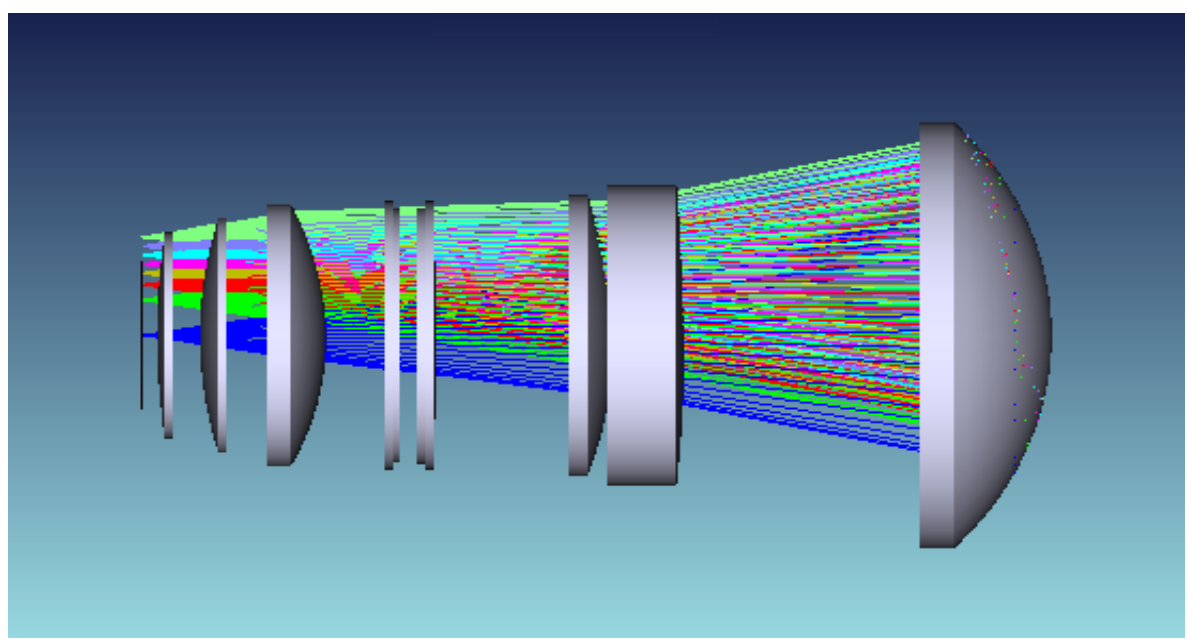

Figure 1: One option (DESpec-SK-3C) for the DESpec optics. From right to left they are C1 to C3, the twocomponent ADC, C4, C5', and the field-flattener C6. The focal plane of fiber-ends would be just to the left of the new C6. "C1" is a little less than 1 meter in diameter. The optical train is 1.9 meters long. $\mathrm{C} 1, \mathrm{C} 2, \mathrm{C} 3$, and $\mathrm{C} 4$ are already installed on the telescope as part of DECam.

\subsection{Optical corrector}

DECam was required to deliver images that were comparable to the median site seeing. For DESpec, images are required to fall within a fiber diameter that is optimized for maximal signal-to-noise $(\mathrm{S} / \mathrm{N})$ ratio of faint galaxies along with a minimum required spectral resolution. While the requirements on image sizes are similar, the impact of degradation in image size is different in the two cases. For DECam, a degradation of image size results in an inability to measure the shapes of the smallest and faintest galaxies. For DESpec, a degradation of image size results in a reduced $\mathrm{S} / \mathrm{N}$ ratio, but one can compensate (within limits) by increased observing time. DECam placed no constraint on the telecentricity of the beam incident on the focal plane, and the incoming beam is tilted up to 4 degrees at the focal plane edge. For DESpec, the fibers are constrained to be perpendicular to the focal plane, and an inclined beam would cause focal ratio degradation at the exit of the fiber. Thus, the beam needs to be perpendicular to the focal plane (telecentric) at all locations.

In addition, the DESpec corrector is required to produce a good image (though not as good as DECam) over the entire useful wavelength range simultaneously. DESpec will reuse the first four elements of the DECam optical corrector (C1C4). As noted above, C5 as well as the DECam filters would not be used for DESpec. For observations at large zenith angles, an Atmospheric Dispersion Compensator (ADC) can compensate for the natural prismatic effect of chromatic refraction in the atmosphere. Because the maximum zenith angle of DESpec has not been finalized, we have developed options for corrector optics both with and without an ADC. Here we present the option with the ADC, and in the next subsection we describe the ADC.

The single C5 lens in DECam is replaced with a pair of lenses, C5' and C6, in DESpec. Both are made of fused silica. Such a pair is needed in order to achieve proper focus and telecentricity simultaneously. One surface (the concave face of the new C5') is aspheric. By using an asphere, the image quality is significantly improved at the field edge, and the curvature of $\mathrm{C} 5$ can be significantly reduced. Both lenses are rather thin, and the presence of an asphere on one might be of some concern. The thinness is somewhat to compensate for the extra glass thickness introduced by the ADC. However, the spectroscopic corrector lens of the SDSS $2.5 \mathrm{~m}$ telescope is even thinner and has a more severe aspheric shape on its convex side, so fabrication is expected to be feasible.

The present default DESpec corrector design, "DESpec-SK-3C," is shown in Figure 1. This design achieves good spot size for wavelengths $500 \mathrm{~nm}<\lambda<1050 \mathrm{~nm}$, as shown in Figure 2. The RMS spot radius is 0.25 arcsec at the center and $0.44 \mathrm{arcsec}$ at the edge. The peak off-incidence ray (non-telecentricity) is at a 0.45 degree angle of incidence. The focal surface has a radius of curvature of $8047.2 \mathrm{~mm}$. The focal ratio of the corrector, $f / 2.9$, is in the optimal range for collecting light in an optical fiber ${ }^{[5]}$. 


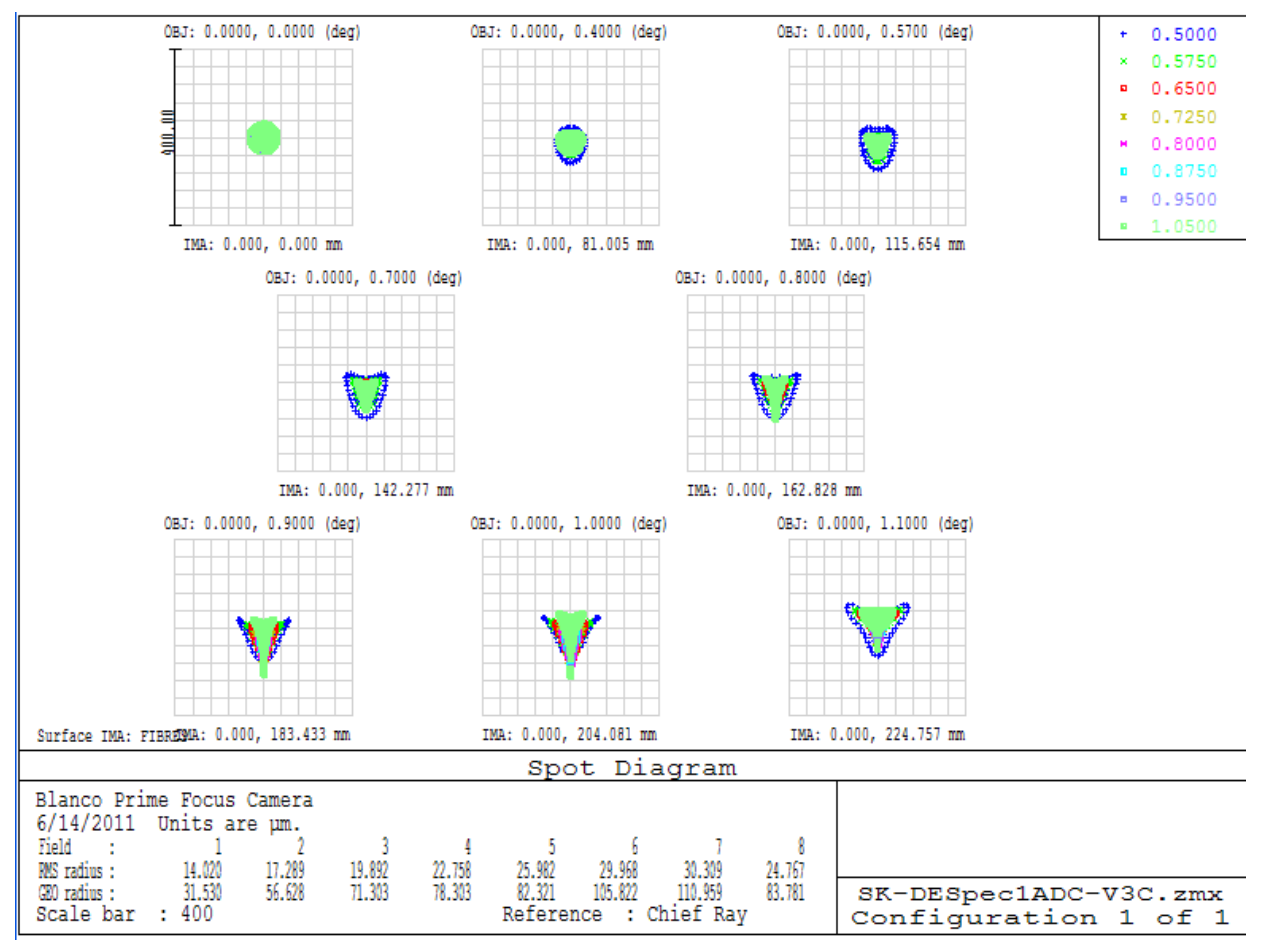

Figure 2: The spot size versus wavelength $(0.5$ (blue) -1.05 (green) microns) from the center of the focal surface (top-left) to the edge of the focal surface (radius of $1.1 \mathrm{deg}$, lower-right). The RMS spot radius is 0.26 arcsec at the center, 0.52 arcsec at the worst radius, and 0.44 arcsec at the edge. These results are from the "DESpec-SK-3C" design.

\subsection{Atmospheric dispersion compensator}

The atmospheric dispersion compensator (ADC) is composed of a pair of crossed Amici prisms. By selecting the rotation angle of the crossed prisms, one can compensate for the dispersive effects of the atmosphere up to zenith angles of 60 degrees. Each prism is itself a double prism made of a crown and a flint glass with similar refractive indices. For the DESpec ADC we have chosen N-BK7 and LLF1, since these glass types have worked well in other large ADCs. For example, the ADC designed for the WIYN One-Degree Imager ${ }^{[6]}$ is similar in size and design to the DESpec ADC. Optical studies show that improved images at the edge of the field can be obtained by making the first and last surfaces of the ADC mildly curved. Such an approach has been used for previous ADCs (e.g., the current Blanco prime focus corrector) and recent conversations with vendors indicate that they are not expected to be difficult to manufacture.

DESpec's ADC will fit into the large slot in the barrel that DECam uses for the filter-changer. There is sufficient room for an ADC in the DECam barrel at the position of the filter-changer/shutter assembly, which is removable or installable in a short time. We do not need to use the filter changer while using the DESpec spectrographs.

\section{DESPEC FIBER POSITIONER}

The fiber positioner should be able to support $\sim 4000$ fibers at prime focus. The fiber positioner must move the tips of the optical fibers to predetermined positions for each exposure and then hold them in place for the length of the exposure. It must also gather the fibers into bundles that optically feed the spectrographs. Since DESpec will reutilize the corrector optics of DECam, the focal plane will be about the same size, i.e., a diameter D $450 \mathrm{~cm}$. This implies a separation between fibers (i.e., the pitch) of $\sim 7 \mathrm{~mm}$. Figure 3 shows the locations of 3781 fiber centers within the clear aperture using a $7 \mathrm{~mm}$ fiber pitch. 


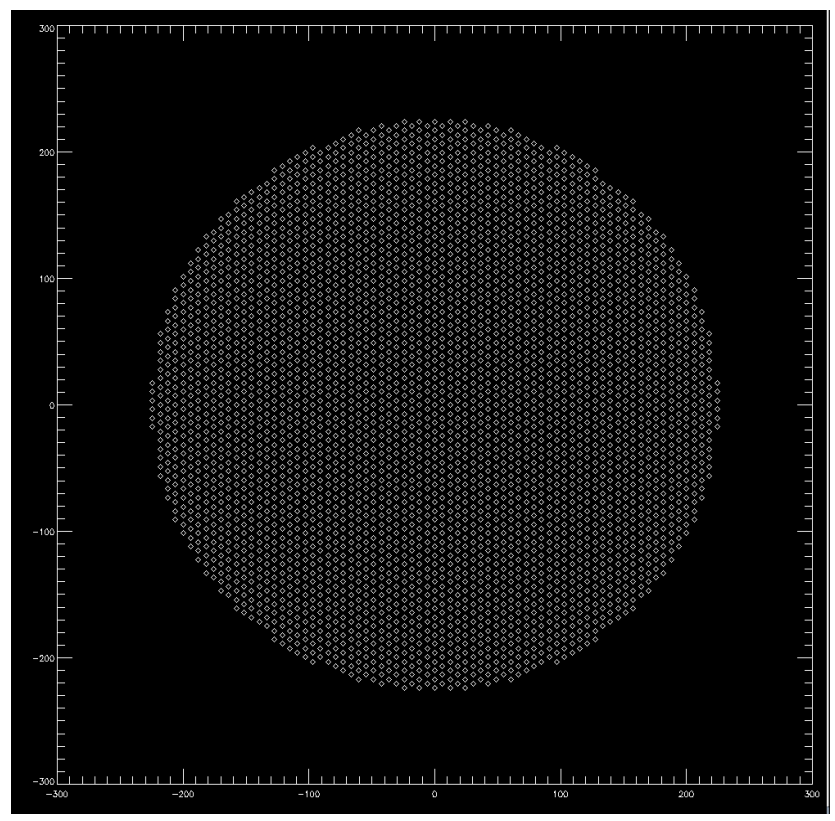

Figure 3: Array of DESpec fiber positioners. Here we show 3781 locations with $7 \mathrm{~mm}$ spacing (pitch) within the clear aperture. If the pitch were $6.3 \mathrm{~mm}$, the clear aperture could accommodate 4675 fiber positioners. The $\mathrm{x}$ and $\mathrm{y}$ axes have units of $\mathrm{mm}$. We have used a clear aperture of $\mathrm{D}=451 \mathrm{~mm}$.

There are two general classes of fiber positioners that could provide technical solutions for DESpec; here we refer to these as the "Twirling Post" design and the "Tilting Spine" design. Figure 4 shows a schematic ${ }^{[7]}$ of the "Cobra" fiber positioner, under development at Caltech/JPL, that was considered for the WFMOS instrument and has been adopted for the Subaru Prime Focus Spectrograph (part of the SuMIRe project). The fiber is held at the tip of the "twirling post." The post can rotate, and the tip of the post can rotate with respect to the post, thus allowing the fiber to be positioned anywhere within a "patrol radius." A variant of this (phi-phi) design has a rotatable post with a tip that moves along the radius (r-phi).
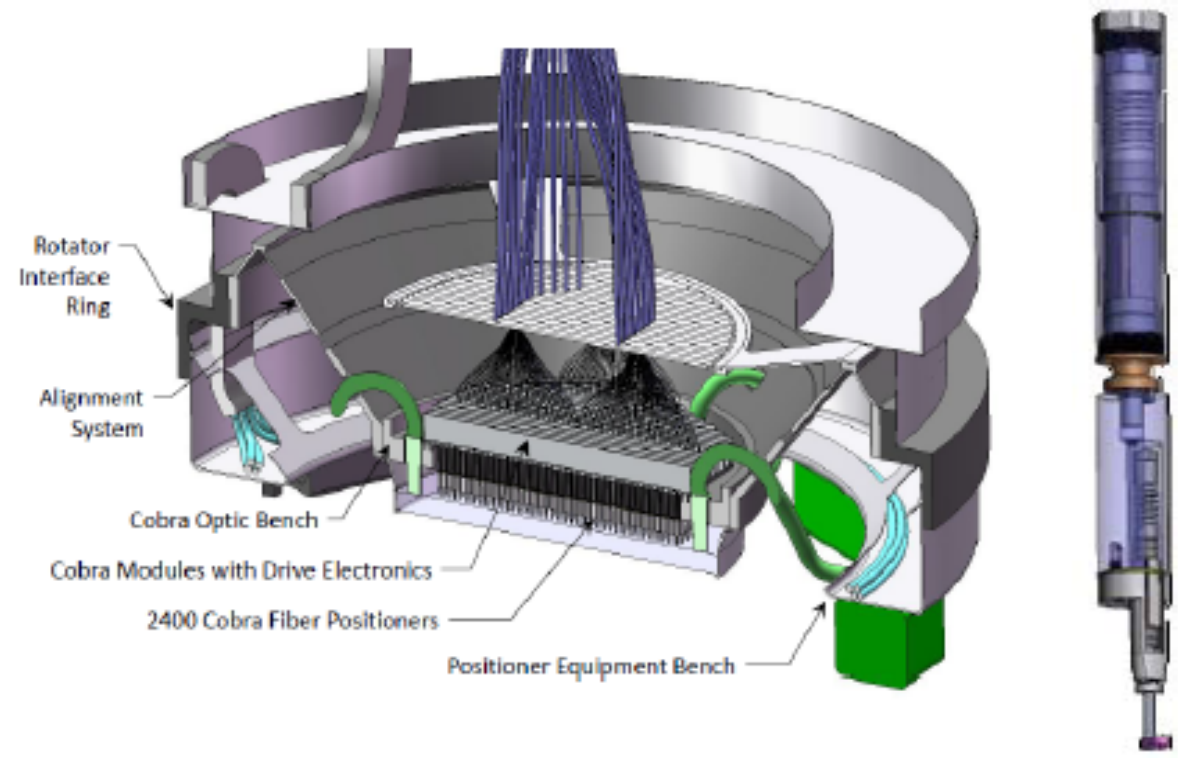

Figure 4: The "Cobra" fiber positioner ${ }^{[8]}$ designed for WFMOS (left). The tip of the fiber is held at the bottom end of the unit positioner (right). 
Figure 5 shows a schematic of an example of the second class of fiber positioner under consideration for DESpec, the "MOHAWK" fiber positioner ${ }^{[9]}$ developed at the Australian Astronomical Observatory. MOHAWK evolved from a previous generation instrument, the "Echidna" fiber positioner ${ }^{[10]}$, which is currently used in the FMOS instrument on the Subaru Telescope in Hawaii. This "Tilting Spine" solution uses a unit positioner (Figure 6) that is tilted into place by a simple piezo-electric actuator at the base.

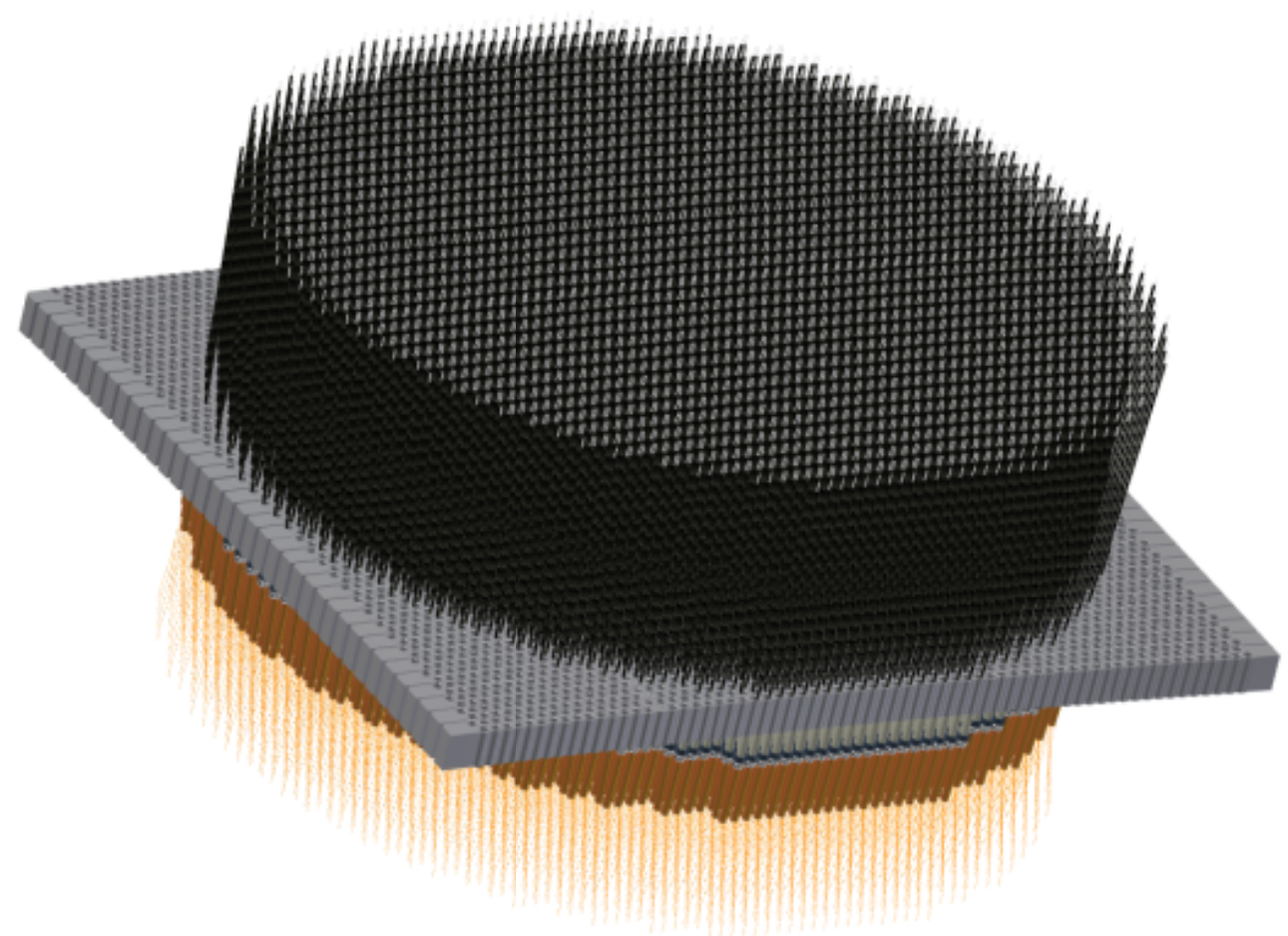

Figure 5: The MOHAWK fiber positioner with DESpec specifications, i.e., 4000 fibers with a $7 \mathrm{~mm}$ pitch, placed in a $450 \mathrm{~mm}$ diameter focal plane.

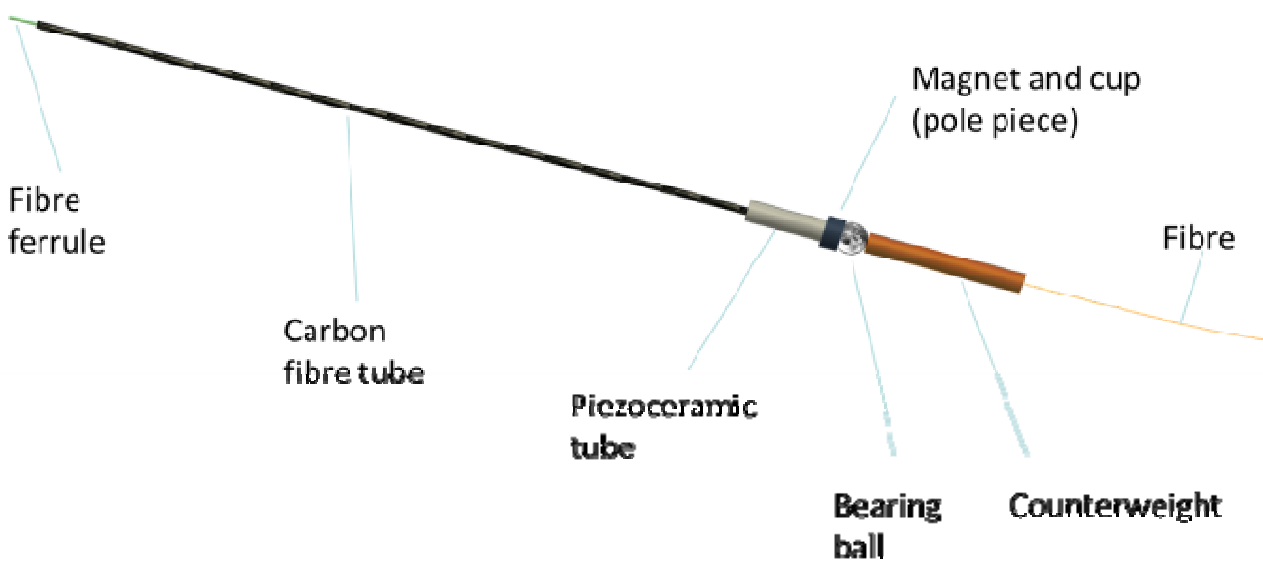

Figure 6: The MOHAWK unit fiber positioner. The spines pivot from mounts at the base and are driven by piezo-electric actuators. 
Figure 7 shows a schematic drawing of a MOHAWK-style fiber positioner as part of the DESpec instrument.

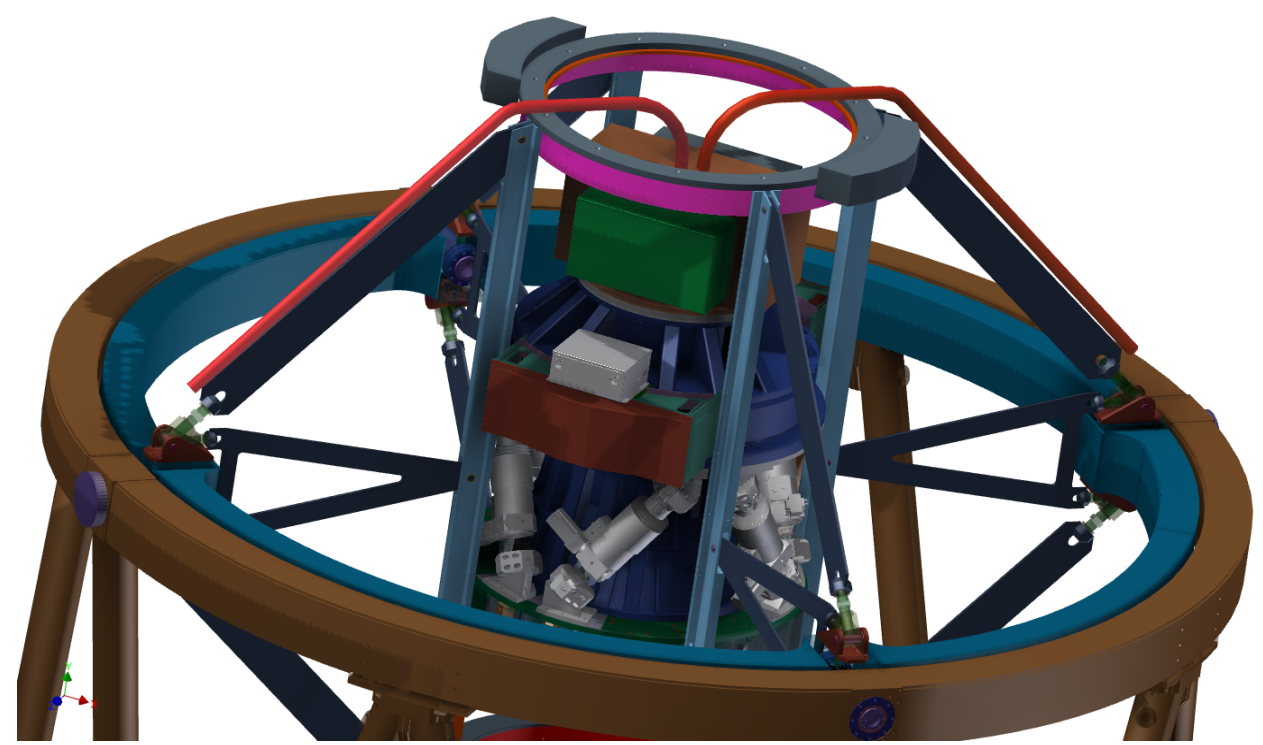

Figure 7: A schematic drawing of a MOHAWK-style fiber positioner positioned on top of the DESpec corrector optics, including the fiber bundles that would feed potential DESpec spectrographs.

At present, the currently operating "Tilting Spine" type positioner (Echidna) allows for a smaller fiber pitch (7mm) than the operating "Twirling Posts" (WFMOS-Cobra prototypes, $12 \mathrm{~mm}$ ). The patrol radius for Echidna is equal to the pitch, and all seven "nearest-neighbor" fibers can be positioned as close together as the size of the tip of the spine allows. The patrol radius for the prototype Twirling Posts is about $2 / 3$ of the pitch and there are not so many nearest-neighbors. A scaled-up Echidna to DESpec size would have 4000 fibers. The Cobra-type positioner would have 1000-1500 fibers with the present state-of-the-art; however, it is expected that in future instruments the pitch could be decreased, which would increase the number of fibers.

Some infrastructure is required to operate the fiber positioner. Any design requires a system to measure the fiber position during configuration so that the position can be verified to be correct within about 10 microns. To position the fibers correctly, the fibers are moved into position and then back-illuminated. Next, a camera is used to check the new position of the fiber. This process is iterated up to 7 times. The total configuration time is expected to be less than the CCD readout time of $\sim 45$ seconds (to achieve $3 \mathrm{e}$-/pixel read noise, see below). Such a fiber back-illumination system was provided within fiber connectors for FMOS, and by illuminating the spectrograph slits in $2 \mathrm{dF}$. Guide and focus capabilities may be accomplished by mounting a small number of CCDs in the DESpec focal plane.

\section{DESPEC UNIT SPECTROGRAPHS AND OPTICAL FIBERS}

A set of multiple identical DESpec unit spectrographs will collect light from the fiber positioners in the focal plane through optical fibers and produce and record science spectra. The key elements driving the optical design of the spectrographs are the wavelength range, the required spectral resolution, and the diameter of the optical fibers carrying the light from the focal surface. To accommodate 4000 fibers, each of 10 spectrographs must accept $\sim 400$ fibers. There are several options for the physical location of the spectrographs: they could be mounted off-telescope or, if sufficiently small and light-weight, they could be mounted near the top of the telescope and arrayed around its upper ring in order to minimize fiber length and thereby increase the throughput of the instrument.

As we are currently continuing to develop the science and survey requirements, we are considering two spectrograph designs. The first design is a single-arm spectrograph with a wavelength range $550<\lambda<950 \mathrm{~nm}$. The second is a two-arm spectrograph in which the blue side has wavelength range $480<\lambda<780 \mathrm{~nm}$ and the red side covers $750<\lambda<1050 \mathrm{~nm}$. 
Both one-arm and two-arm spectrograph designs have sufficient resolution to resolve the redshifted $3727 \AA$ OII doublet, which requires $\mathrm{R}>1200$. Both spectrographs also have high enough resolution to minimize contamination of the science spectrum by night sky lines in the red, which requires $\mathrm{R}>2000^{[11]}$.

\subsection{Single-Arm Spectrographs}

The DESpec unit single-arm spectrograph optical design is conceptually based on VIRUS, the instrument currently being built for the HETDEX survey ${ }^{[12]}$ that will be carried out with the Hobby-Eberly Telescope. HETDEX requires at least 150 and can accommodate up to 192 of these spectrographs, so they must be inexpensive and simple to produce in bulk. The VIRUS unit spectrograph design ${ }^{[13],[14]}$ is a high-throughput, single-arm blue spectrograph. Figure 8 shows the optical layout of VIRUS, which can be easily optimized for the DESpec wavelength regime and spectral resolution.

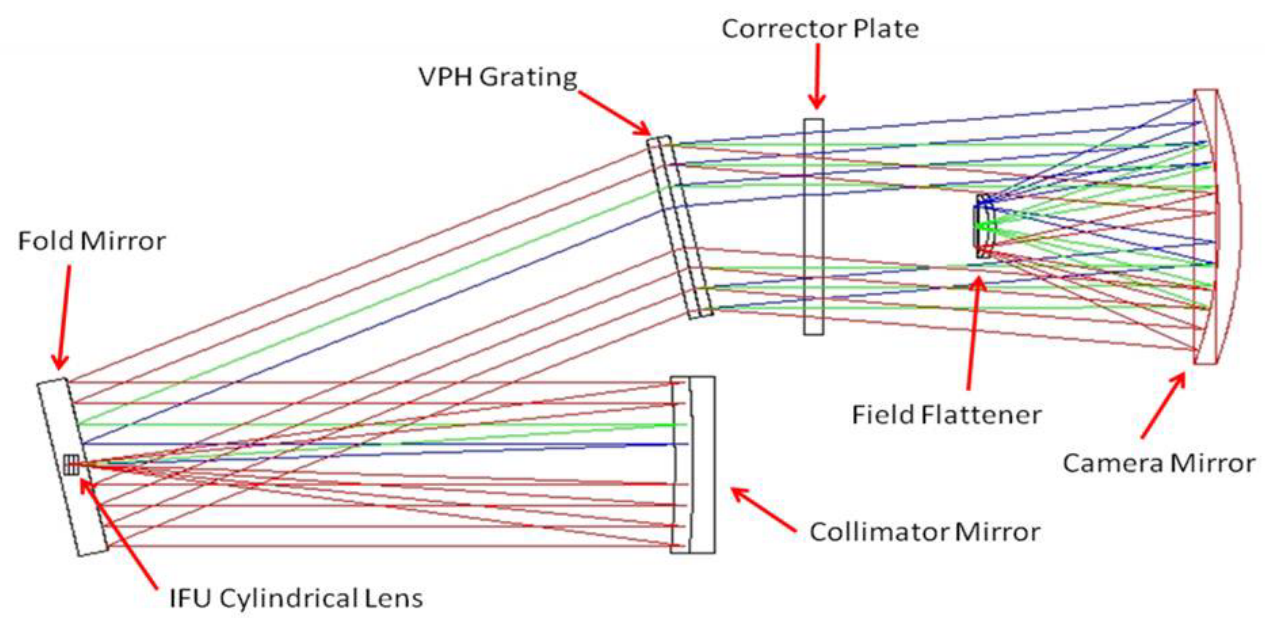

Figure 8: Optical layout of one of the VIRUS unit spectrographs. Light enters through a fiber slit on the left and is reflected by the collimator mirror and fold mirror before it passes through the grating. The vacuum Schmidt camera houses the CCD detector, which is located in the middle of the optical beam, just to the left of the field flattener lens. The overall length of the VIRUS is $\sim 0.75 \mathrm{~m}$.

The VIRUS instruments are composed of a main spectrograph body, which houses the collimator optics and the disperser, and a vacuum vessel that houses a Schmidt-type camera. A volume phase holographic (VPH) grating acts as the dispersing element. The collimator unit consists of a spherical collimator mirror, folding flat mirror, and the VPH grating. The Schmidt camera consists of an aspheric corrector lens that serves as the Dewar window, a spherical camera primary mirror, an aspheric field flattener lens, and a CCD detector that is held in the center of the camera on a one-arm "spider" assembly.

DESpec, like VIRUS, would be a set of simple, fixed spectrographs with no moving parts. This feature of the instrument makes it inexpensive, quick to assemble, and easy to mount on or near the telescope.

In the designs presented here, we assume the use of $100 \mu \mathrm{m}$ diameter $(1.75 \operatorname{arcsec})$ fibers with a 2.6 pixel/fiber resolution element (see discussion in Section 4.3). These parameters require an $f / 1.3$ camera. For comparison the HETDEX VIRUS camera is $f / 1.33$. In these calculations we assume an $f / 2.75$ input to the spectrograph and account for the fact that the fibers are round rather than square (which effectively increases the resolution by a factor of 1.2). We would use 20 DECam red-sensitive, fully-depleted CCDs, two for each camera focal plane, laid out in a 4k x 4k array. Each spectrograph would accept light from 400 fibers (200 per CCD) with spectra along the 4k direction. Table 1 lists some of the parameters for a potential single-arm spectrograph design. The resolution of the single-arm spectrograph is $\mathrm{R}=\lambda / \Delta \lambda \sim 2850$ at $750 \mathrm{~nm}$. The exact wavelength coverage of this spectrograph could easily be shifted by up to $50 \mathrm{~nm}$. 


\begin{tabular}{|l|l|}
\hline Parameter & Single-Arm Spectrograph \\
\hline Fiber diameter & $100 \mu \mathrm{m}(1.75 \mathrm{arcsec})$ \\
\hline Wavelength range $(\mathbf{n m})$ & $550<\lambda<950$ \\
\hline CCD & $\mathrm{DECam} 2 \mathrm{kx} 4 \mathrm{k}$ \\
\hline Resolution $(\Delta \lambda)$ & $0.263 \mathrm{~nm}$ \\
\hline \# pixels/fiber & 2.6 \\
\hline Spectral resolution & $\mathrm{R} \sim 2850$ at $750 \mathrm{~nm}$ \\
\hline Camera $f$ /\# & $f / 1.3$ \\
\hline Camera type & Reflective \\
\hline
\end{tabular}

Table 1: Parameters for a potential one-arm spectrograph design.

\subsection{Two-Arm Spectrographs}

The two-arm spectrograph design would enable an increase in the wavelength range and spectral resolution, with the cost of increased complexity. It uses a refractive camera for each arm. Parameters for a two-arm spectrograph covering the wavelength range $480<\lambda<1050 \mathrm{~nm}$ are shown in Table 2 . The spectral resolution of the blue arm is $\mathrm{R}=\lambda / \Delta \lambda \sim 2760$ at $630 \mathrm{~nm}$; the red arm has R 3950 at $900 \mathrm{~nm}$.

\begin{tabular}{|l|l|l|}
\hline Parameter & Blue Arm & Red Arm \\
\hline Fiber diameter & \multicolumn{1}{|c|}{$100 \mu \mathrm{m}(1.75 \mathrm{arcsec})$} \\
\hline Wavelength range $(\mathbf{n m})$ & $480<\lambda<780$ & $750<\lambda<1050$ \\
\hline CCD & $\begin{array}{l}\text { Blue-sensitive } \\
2 \mathrm{kx} 4 \mathrm{k}\end{array}$ & DECam 2kx4k \\
\hline Resolution $(\boldsymbol{\Delta} \lambda)$ & $0.228 \mathrm{~nm}$ & $0.228 \mathrm{~nm}$ \\
\hline \# pixels/fiber & 3 & 3 \\
\hline Spectral resolution & $\mathrm{R} \sim 2760$ at $630 \mathrm{~nm}$ & $\mathrm{R} \sim 3950 @ 900 \mathrm{~nm}$ \\
\hline Camera $\boldsymbol{f} / \#$ & $f / 1.5$ & $f / 1.5$ \\
\hline Camera type & \multicolumn{2}{|c|}{ Refractive } \\
\hline
\end{tabular}

Table 2: Parameters for a potential two-arm spectrograph design.

An example of a spectrograph of approximately this design was proposed for WFMOS ${ }^{[15]}$. It is a high-throughput, 2-arm spectrograph with all-refractive optics and VPH gratings. Another example of this type of design is the conceptual design of the GMACS wide-field, multi-object optical spectrograph ${ }^{[16]}$ for the Giant Magellan Telescope. GMACS is a set of four fully refractive spectrographs divided into blue and red channels using a dichroic. Conceptually, the DESpec spectrographs could be scaled down versions of the WFMOS or GMACS spectrographs. The benefit of an all refractive, two-arm spectrograph design is the maximal throughput of the system (i.e., the science beam is not occulted by a CCD in the camera), the increased wavelength coverage, and the potential for higher spectral resolution.

\subsection{Optical Fibers}

The light is carried from the fiber positioner to the spectrographs with optical fibers. The diameter of the fibers depends on the expected source flux distribution and the sky background and should be chosen to maximize the $\mathrm{S} / \mathrm{N}$ ratio of a sky-dominated object spectrum. In this section we present initial results from a study that optimizes fiber diameter for a flux-limited survey in the presence of a dominant sky background. Note however that the DESpec fiber diameter could change as the science case evolves.

The calculations start with a magnitude limit and compute the total number of galaxies per square degree at this limit. Next a fiber diameter is chosen, and the rate at which spectra are collected to a pre-determined $\mathrm{S} / \mathrm{N}$ ratio as a function of galaxy magnitude and radius is calculated. The distribution of galaxy radii and magnitudes comes from the COSMOS 
simulation $^{[17]}$. Because large galaxies tend to have low surface brightness, they are the most difficult spectroscopic targets. The spectra collection rates are calculated using a weighted average over a distribution of CTIO-measured seeing. A contribution of 0.6 arcsec from the optics PSF is included. For magnitude limits in the range 22 to 24 , the optimal fiber size is in the range 1.8 to 2.0 arcsec. However, the rate at which redshifts are collected depends only weakly on fiber size: the range in fiber sizes that have success rates within $10 \%$ of the peak rate correspond to diameters ranging from 1.5 to 2.4 arcsec. The intersection of these ranges that is near-optimal for all limiting magnitudes is 1.7 to 2.1 arcsec. Because the optimization has determined that the fiber diameter is a soft minimum, we expect that the final diameter can be selected based largely on other considerations, such as availability of off-the-shelf fiber sizes, spectrograph resolution, or fiber positioning accuracy. For now we have assumed standard 100 micron fibers, which correspond to a 1.75 arcsec diameter at the focal plane.

The input $f$-ratio of the fibers is $f / 2.9$, which is well-matched to the acceptance angle of optical fibers and results in high throughput and low focal ratio degradation ${ }^{[18]}$. The length of the fibers is 10 to 30 meters and depends on where the spectrographs are mounted on the telescope; obviously we will minimize the length of the fibers in order to increase throughput of the instrument. Figure 9 shows fiber throughput as a function of fiber length for six wavelengths from 500 to $1100 \mathrm{~nm}$ in a Polymicro FBP fiber. Finally, we note that several fibers per spectrograph will point at blank sky to measure the sky spectrum for sky subtraction.

Polymicro FBP fiber transmission

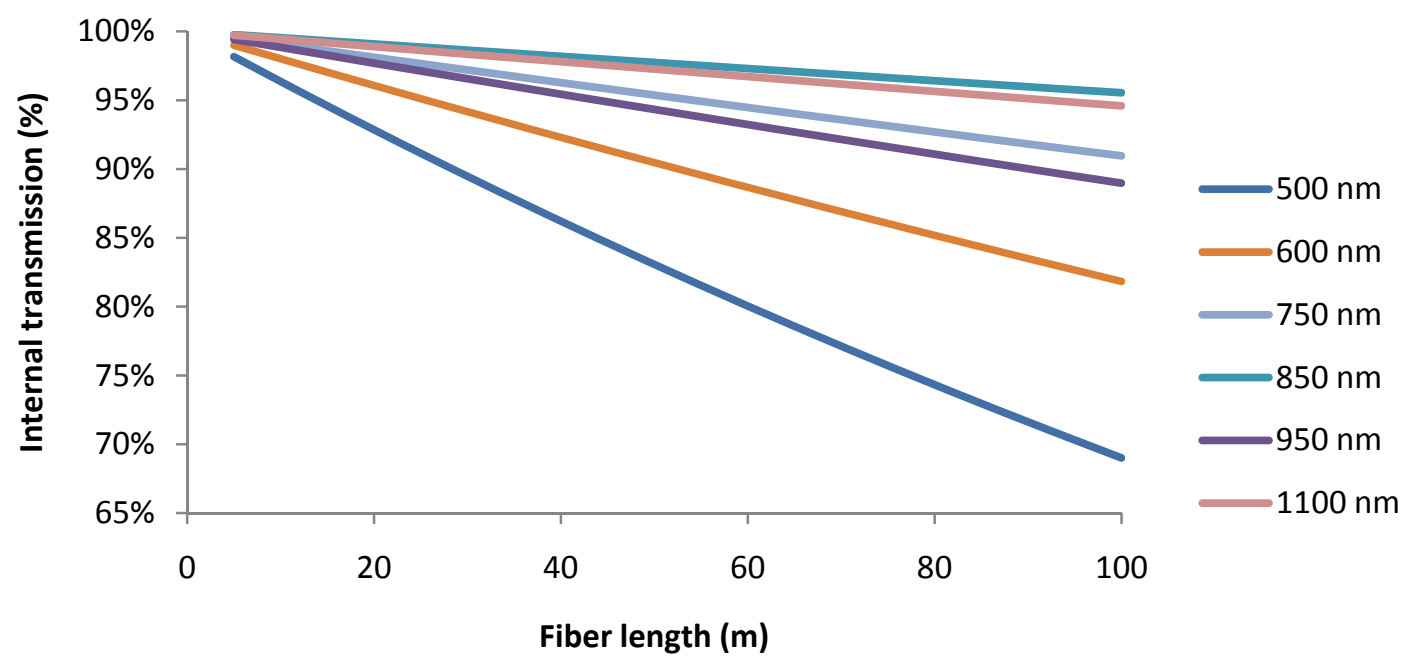

Figure 9: Fiber throughput as a function of fiber length at six different wavelengths for Polymicro FBP broadband fibers. This assumes optimal f-number, which is $f / 3$ to $f / 4$. Note that at shorter wavelengths the fibers have a lower throughput, which gets progressively worse for $\lambda<500 \mathrm{~nm}$. Data provided by Polymicro.

\section{DESPEC DETECTORS}

The DESpec unit spectrographs will produce spectra that are recorded by CCD detectors. DESpec will use $2 \mathrm{k} \times 4 \mathrm{k}$ backside-illuminated, red-sensitive CCDs designed by LBNL, for either the one-arm spectrograph or for the red arm of the two-arm spectrograph. These CCDs have high quantum efficiency $(\mathrm{QE})$ at near infrared wavelengths. They are 250 microns thick and attain good ( $\sim 5$ micron) dispersion characteristics from a $40 \mathrm{~V}$ substrate bias. The 4-side buttable CCD package is suitable, so existing spare, tested, packaged, science-grade DECam CCDs ${ }^{[19]}$ can be used on DESpec, providing a significant cost savings. Figure 10 shows the QE of three CCDs produced for DECam. 


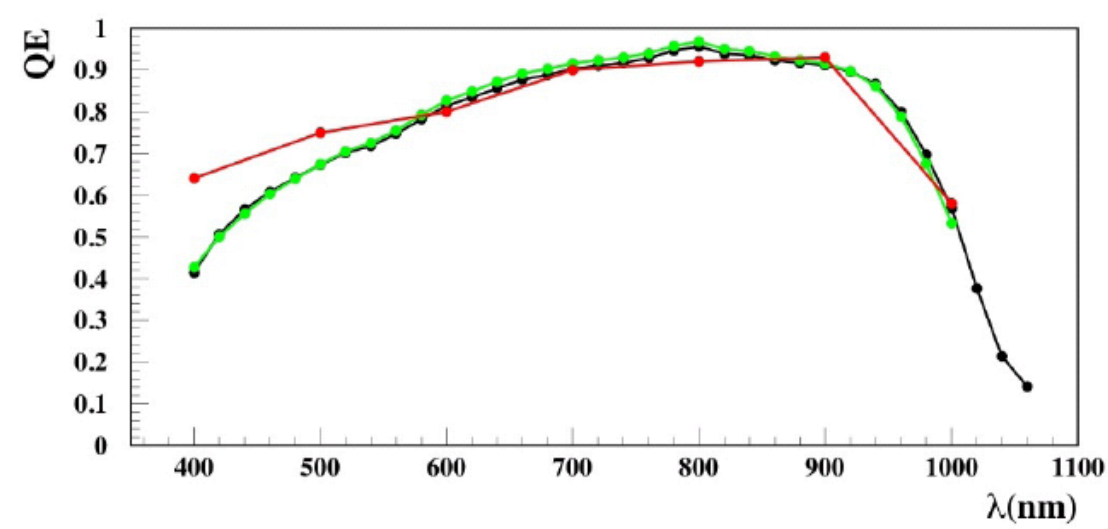

Figure 10: The absolute QE of three typical CCDs produced for the Dark Energy Camera. The blue side of the two-arm spectrographs could also use DECam 2kx4k CCDs.

The DECam CCD electronics can read out the $2 \mathrm{kx} 4 \mathrm{k}$ CCDs in $\sim 17$ seconds with $\sim 10$ electron/pixel read noise (for the DES imaging survey). These devices can also be read out in 45 seconds with only 3 electron/pixel read noise. These detectors satisfy the DESpec requirements. Ongoing development at Fermilab on low read noise techniques may reduce the read noise to well under 1 electron/pixel, should that be desirable.

The blue arm of the two-arm spectrograph design will use commercially available $2 \mathrm{k} x 4 \mathrm{k}$ blue-sensitive CCD detectors, likely with similar readout electronics to those used in the DECam CCDs.

\section{INTERCHANGEABILITY WITH DECAM}

The Dark Energy Camera has been designed for efficient installation and removal from the CTIO Blanco telescope Prime Focus Cage. Figure 11 shows the DECam camera installation fixture positioned in front of the Prime Focus Cage, mounted on the Blanco Telescope Simulator at Fermilab ${ }^{[20]}$. To change from DECam to DESpec, one would tilt the telescope to the northwest platform and use the DECam camera installation fixture to remove DECam. DECam is then stowed off of the telescope with its Dewar window, the camera's final optical element (C5), in place. DESpec, which will have been stowed either off-telescope or on the telescope structure, is connected to a similar installation fixture for inserting into the cage.
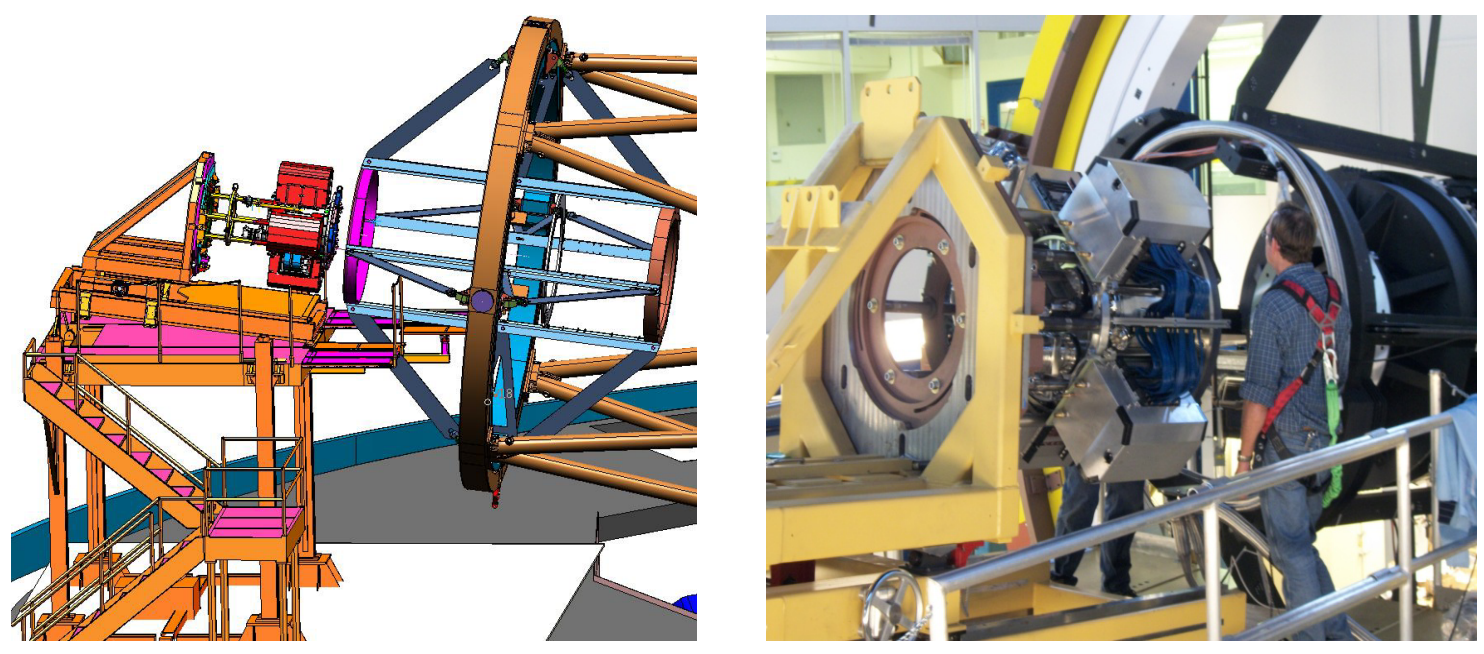

Figure 11: The DECam camera installation fixture (schematic at left, in close-up at right) at Fermilab. Right image shows the installation fixture being used to mount the camera in the Prime Focus Cage (black, at right). 


\section{THE BLANCO TELESCOPE}

The Blanco $4 \mathrm{~m}$ telescope at Cerro-Tololo Inter-American Observatory (CTIO) is currently home to the DECam instrument. The telescope has been in use since 1974 and has been host to dozens of successful astronomical instruments. An extensive set of improvements made a decade ago included replacement of the passive primary mirror supports with an active system and alterations to the telescope environment to improve the air flow and remove heat sources. More recent improvements made prior to the installation of the Dark Energy Camera include replacement of the primary mirror's radial supports, which has substantially reduced motion of the primary in its cell, and an upgrade of the telescope control system. Figure 12 shows a photograph of the Blanco 4m telescope with the new DECam prime focus cage, installed on the telescope in May 2012.

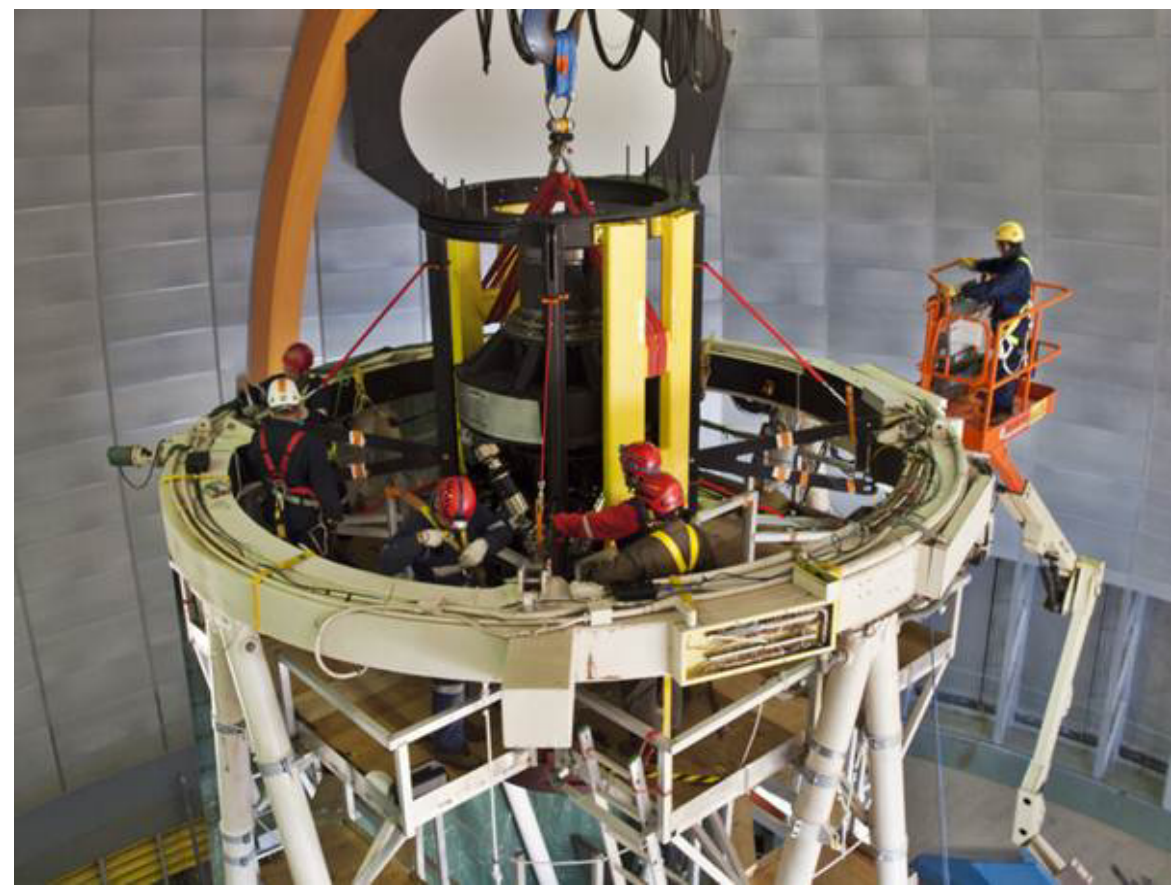

Figure 12: The new top end of the Blanco $4 \mathrm{~m}$ telescope, during installation of the new prime focus cage to hold DECam.

\section{CONCLUSIONS}

We have described DESpec, a potential multi-fiber instrument for the CTIO Blanco $4 \mathrm{~m}$ telescope. The instrument could be used to provide massive spectroscopic follow-up of dark energy sources discovered in the DES and LSST imaging surveys, and could be a CTIO facility instrument to be used for multiple science projects. We have presented technical solutions for the DESpec instrument design and have demonstrated the feasibility of such an instrument. The instrument design will continue to evolve along with the science case for the project. As a result, the instrument design described here does not represent final technical choices; however, it does represent feasible solutions that could be implemented at low risk and low cost were the project to move forward.

\section{ACKNOWLEDGEMENTS}

Texas A\&M University thanks Charles R. '62 and Judith G. Munnerlyn, George P. '40 and Cynthia Woods Mitchell, and their families for their support of astronomical instrumentation activities in the Department of Physics and Astronomy.

Fermilab is operated by Fermi Research Alliance, LLC under Contract No. De-AC02-07CH11359 with the United States Department of Energy. 


\section{REFERENCES}

[1] Flaugher, B., et al., "Status of the Dark Energy Camera (DECam) Project," Proc. SPIE 8446, 35 (2012).

[2] Diehl, H. T., et al., "The Dark Energy Survey Camera (DECam)," Proc. Of the $2^{\text {nd }}$ International Conference on Technology and Instrumentation in Particle Physics, in print (2012).

[3] Albrecht, A., et al., "Report of the Dark Energy Task Force," astro-ph/0609591(2006).

[4] Schlegel, D., et al., "The BigBOSS Experiment," arXiv:1106.1706 (2011).

[5] Ramsey, L. "Focal ratio degradation in optical fibers of astronomical interest" ASPC Conf. Proc.: Fiber Optics in Astronomy, Tucson, AZ, 26-39 (1988).

[6] Muller, G. P. "Mechanical design of the WIYN One Degree Imager (ODI)," Proc. SPIE 7014, 156 (2008).

[7] Braun, D., et al., "Developments in high density Cobra fiber positioners for the Subaru Telescope's Prime Focus Spectrometer," Proc. SPIE 8450, 41 (2012).

[8] Seiffert, M. presentation at Princeton University, http://www.astro.princeton.edu/astroevents/widefieldsurveys/wide_field/seiffert_princeton.pdf (2009).

[9] Saunders, W., et al. "MOHAWK: a 4000 fiber positioner for DESpec," Proc. SPIE 8446, 188 (2012).

[10] Akiyama, M., et al. "Performance of Echidna fiber positioner for FMOS on Subaru," Proc. SPIE 7018, 94 (2008).

[11] Villanueva, S., Jr., DePoy, D. L., Marshall, J. L., "Optimal resolutions for optical and NIR spectroscopy," Proc. SPIE 8446, 105 (2012).

[12] Hill, G. J., et al., "The Hobby-Eberly Telescope Dark Energy Experiment (HETDEX): Description and Early Pilot Survey Results," ASPC Conf. Proc.: Panoramic Views of Galaxy Formation and Evolution, 399, 115 (2008).

[13] Hill, G. J., et al., "VIRUS: a massively replicated 33k fiber integral field spectrograph for the upgraded HobbyEberly Telescope," Proc. SPIE 7735, 21 (2010).

[14] Marshall, J. L., et al. "Production-line assembly of 150+ VIRUS spectrographs," Proc. SPIE 7735, 152 (2010).

[15] Smee, S., et al., "Design of a multi-object high-throughput low-resolution fiber spectrograph for WFMOS," Proc. SPIE, 6269, 78 (2006).

[16] DePoy, D. L., et al, "The GMACS Spectrograph for the Giant Magellan Telescope," Proc. SPIE 8446, 58 (2012).

[17] Jouvel, S., et al., "Designing future dark energy space missions. I. Building realistic galaxy spectro-photometric catalogs and their first applications," A\&A, 504, 359 (2009).

[18] Murphy, J. D., et al., "Focal ratio degradation and transmission in VIRUS-P optical fibers," Proc. SPIE 7018, 92 (2008).

[19] Estrada, J., et al., "Focal plane detectors for Dark Energy Camera (DECam)," Proc. SPIE 7735, 59 (2010).

[20] Diehl, H. T., et al., "Testing the Dark Energy Camera on a telescope simulator," Proc. SPIE 7735, 119 (2010). 